\title{
Membrane of Crista
}

National Cancer Institute

\section{Source}

National Cancer Institute. Membrane of Crista. NCI Thesaurus. Code C33078.

The part of the inner mitochondrial membrane that folds inward to enclose the cristae. 